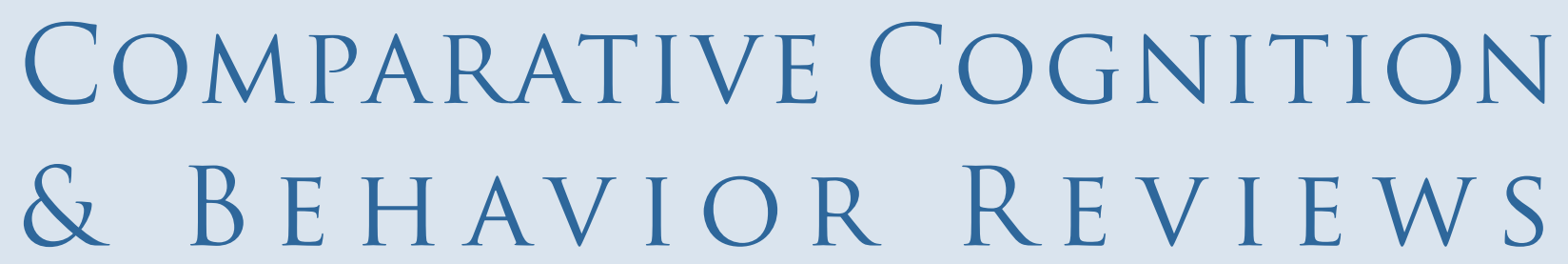

\title{
Functional Magnetic Resonance Imaging of the Domestic Dog: Research, Methodology, and Conceptual Issues
}

\author{
Andie M. Thompkins \\ Dept. of Psychology, Auburn \\ University, Auburn, AL, USA
}

\author{
Gopikrishna Deshpande \\ Dept. of Psychology, Auburn \\ University, Auburn, AL, USA \\ AU MRI Research Center, \\ Dept. of Electrical \& Computer \\ Engineering, Auburn University, \\ Auburn, AL, USA \\ Alabama Advanced Imaging \\ Consortium, Auburn University \\ and University of Alabama \\ Birmingham, AL, USA
}

\author{
Paul Waggoner \\ Canine Performance Sciences, \\ College of Veterinary Medicine, \\ Auburn University, \\ Auburn, AL, USA
}

\author{
Jeffrey S. Katz \\ Dept. of Psychology, Auburn \\ University, Auburn, AL, USA \\ AU MRI Research Center, \\ Dept. of Electrical \& Computer \\ Engineering, Auburn University, \\ Auburn, AL, USA \\ Alabama Advanced Imaging \\ Consortium, Auburn University \\ and University of Alabama \\ Birmingham, AL, USA
}

Neuroimaging of the domestic dog is a rapidly expanding research topic in terms of the cognitive domains being investigated. Because dogs have shared both a physical and social world with humans for thousands of years, they provide a unique and socially relevant means of investigating a variety of shared human and canine psychological phenomena. Additionally, their trainability allows for neuroimaging to be carried out noninvasively in an awake and unrestrained state. In this review, a brief overview of functional magnetic resonance imaging (fMRI) is followed by an analysis of recent research with dogs using fMRI. Methodological and conceptual concerns found across multiple studies are raised, and solutions to these issues are suggested. With the research capabilities brought by canine functional imaging, findings may improve our understanding of canine cognitive processes, identify neural correlates of behavioral traits, and provide early-life selection measures for dogs in working roles.

\section{Keywords: canine fMRI, dog cognition, dog neuroimaging}

\section{Introduction}

The domestic dog (Canis familiaris) has become one of the primary subjects of recent comparative cognition research. The "rise of the dog" is unsurprising given its foundations. Bensky, Gosling, and Sinn (2013) discussed these foundations in a comprehensive review, highlighting the interspecific communication and relationship characteristic of dog-human cohabitation, dogs as models for human cognitive deficiencies, the trainability and availability of dog subjects, and a steadfast interest in dog cognition by the general public. The authors cite exponential growth in the number of dog cognition publications, covering an expanse of sensory modalities, research questions, and dog ages and populations. A more recent development in the rise of the dogs has been the comparative neuroimaging of awake dogs. In this review, we summarize the extant literature on functional magnetic resonance imaging (fMRI) and discuss its implications for comparative research and application. 
The inclusion of dogs in comparative neuroimaging studies has come about due to an ideal combination of scientific relevance and training protocols. Due to the evolutionary history that humans and dogs have shared over tens of thousands of years, dog subjects and human participants enter research with similar environmental experiences and an extensive interspecific social repertoire (e.g., sharing daily environments and companions). Further, the social bond shared between humans and dogs and the corresponding receptivity of dogs to human cues helps alleviate the need for restraint and sedation in neuroimaging studies. Rather, dogs can be trained to lie motionless and awake for neuroimaging scans. Though the dog fMRI literature is in its infancy, lagging far behind the human fMRI literature in scope and number, the unique challenges of this field (e.g., training time, parameter selection) become surmountable when data acquisition and analysis techniques are sophisticated enough to compensate accordingly. Consequently, canine fMRI may require methodological advancements over and above the state-of-the-art in human fMRI for addressing these unique challenges.

The advent of fMRI technology in the 1990s presented the scientific and medical communities with a safe and noninvasive means of imaging brain activity with high spatial resolution. FMRI makes use of the activity-dependent flow of oxygenated blood in order to localize mental functions to specific structures in the brain. That is, when neurons in the brain are activated, an increased volume of oxygenated blood flows to the region in which they are located in order to meet the localized energy demand by the neurons. The volume of oxygen in this blood exceeds what is consumed by the neuronal activity, and therefore a surplus of oxygenated blood in that region gives rise to a localized decrease in magnetic susceptibility (as oxyhemoglobin is

Author Note: Correspondence may be addressed to: Andie M. Thompkins,email: andie.thompkins@auburn.edu; Gopikrishna Deshpande, email: gopi@auburn.edu; Paul Waggoner, email: waggolp@auburn.edu; orJeffreyS.Katz,email:katzjef@auburn.edu.

Acknowledgments: We wish to thank Jennifer Robinson, Lewis Barker, and Ana Franco-Watkins for their considerate feedback in the development of this review. Writing of this review was supported by Defense Advanced Research Projects Agency (government grant/contract number W911QX-13-C-0123). The views, opinions, and/or findings contained in this article are those of the authors and should not be interpreted as representing the official views or policies, either expressed or implied, of the Defense Advanced Research Projects Agency, U.S. Department of Defense, or the federal Government of the United States of America. diamagnetic) and a concomitant increase in MR signal intensity. Thus, the signal used in fMRI is referred to as blood oxygenation level dependent (BOLD) signal (Ogawa, Lee, Kay, \& Tank, 1990).

The increase in blood flow that occurs subsequent to a period of neuronal activity is called the hemodynamic response. In humans, this response temporally lags in comparison to the neuronal activity, reaching its maximum level approximately five seconds after neuronal activity, which may have occurred over the course of milliseconds. Subsequent to this hemodynamic response peak is an undershoot period, by which the signal does not return to baseline until 15 to 20 seconds after its peak. The course of the hemodynamic response (from rise, to peak, to fall, and return to baseline) to an external stimulus is referred to as the hemodynamic response function (HRF). The convolution of the external stimulus and the HRF represents the expected signal in brain regions activated by the external stimulus. By matching this expectation with the measured response using a linear mathematical model, brain regions subserving the processing of the external stimulus can be pinpointed (Ogawa et al., 1992; Poldrack, Mumford, \& Nichols, 2011).

Comparatively, the HRF will differ across species due to differences in vasculature necessitated by varying brain size and shape. For example, in awake rodents, the latency of the HRF peak has been shown to be 2 seconds (Martin, Martindale, Berwick, \& Mayhew, 2006), while the canonical HRF used in humans has a peak latency of 6s (Henson, 2004). The precise shape of the HRF has not been determined in canines, and this is an important limitation for future research to address to further validate canine fMRI. Until such advances have been made, it is reasonable to use time and dispersion derivatives in the general linear activation analysis so as to explicitly model and regress out the variability of the HRF (with respect to the canonical HRF) in experimental data (Jia, Hu, \& Deshpande, 2014).

In order to measure the BOLD response, several magnetic resonance imaging components must come together. In MR technology, a strong static magnetic field serves to align the protons in the body. Emission of radio frequencies is used to intermittently disrupt this alignment of protons, after which the protons realign with the static magnetic field while necessarily emitting energy. This resonance energy is picked up by receiving coils and creates the signal by which fMRI data are obtained (Smith, 2010).

While our emphasis in this review is on fMRI, MRI also allows for detailed structural scans of the brain. The use of MRI for investigating anatomical structure of canine brains predates the use of fMRI for investing canine brain function. The main reason for this is that anatomical MRI 
could be performed on anesthetized dogs without any loss of information since anesthesia does not (at least immediately) alter structure. Hence, MRI has become an invaluable tool in the veterinary field and has been used primarily for clinical purposes (e.g., degenerative diseases, cognitive dysfunction, herniation). There have also been specific applications (e.g., aging, tractography) for domestic dogs based on the anatomical/white-matter structure and development of the brain (e.g., Anaya García, Hernández Anaya, Marrufo Meléndez, Velázquez Ramírez, \& Palacios Aguiar, 2015; Baxi et al., submitted; Gross, Garcia-Tapia, Riedesel, Ellinwood, \& Jens, 2010; Su et al., 2005; Jacqmot et al., 2013).

As interest has risen for assessing nonhuman cognition via functional MRI studies, a growing variety of species have been imaged in MR scanners. The current driving force of progress in the expansion of fMRI research stems from the possibility of keeping animals in a still, wakeful, and attentive state during scanning. Experimental and training techniques to allow for awake scanning have been developed for rats (King et al., 2005; Lahti, Ferris, Li, Sotak, \& King, 1998), pigeons (De Groof et al., 2013), monkeys (e.g., Chen, Wang, \& Dillenburger, 2012) and dogs (e.g., Jia, Pustovyy, et al., 2014). In rats, Lahti et al. (1998) used fMRI to localize somatosensory cortex activation upon shock, and King et al. (2005) furthered methodological development by investigating the effects of experiment acclimation on stress levels. De Groof et al. (2013) used traditional and resting state fMRI to investigate visual system connectivity in awake pigeons. In monkeys, fMRI has been used to explore visual area activation as well as protocol and parameter adjustments for improving image quality. As we will review in detail, Jia et al. (2015) used awake dog subjects to uncover olfaction-driven activations. The key difference with dogs, though, is that, unlike other animals, they do not have to be restrained and can be trained to hold their head still as humans do, making the experiment more valid for comparisons to humans.

Although various training techniques have not been systematically explored, an overarching goal of any training methodology is to reduce training time while maintaining success in behavior. When training dogs to lie still for fMRI, researchers have used a variety of techniques including chaining (e.g., Berns, Brooks, \& Spivak, 2013), target stick (e.g., Jia, Pustovyy, et al., 2014), and model-rival (e.g., Andics, Gácsi, Faragó, Kis, \& Miklósi, 2014) methods. In most cases, training builds incrementally from basic contingencies outside the scanner room (e.g., head on chin rest in mock coil, touching nose to target) to inside the scanner room (e.g., prone position on scanner bed) to inside the scanner bore (e.g., head still in coil for scan). Regardless of training method used, comparisons cannot be made across the current body of literature because the availability of the dogs and handlers to participate in training has varied significantly. Pragmatically, developing techniques that promote rapid acclimation to the scanner environment with minimal stress to the dogs is ideal.

\section{History of fMRI in the Dog}

The use of fMRI provides an exciting and fairly unchartered area of comparative cognition and neuroimaging research with domestic dogs. Explorations in dog MRI and fMRI began with the use of sedation to answer questions about anatomy and physiology, primarily for the purposes of veterinary education and research. Such studies have provided knowledge of canine neural responsiveness, cognitive effects of aging, neuroimaging efficacy, and health viability, and thus we first discuss the work done with anesthetized dogs in this paragraph. Bach et al. (2013) used fMRI to successfully identify neural regions associated with processing of auditory stimuli, as well as establish the efficacy of fMRI with anesthetized dogs in regard to auditory stimulus presentation. Su et al. (2005) used longitudinal structural MRI to investigate the time course of neural correlates of canine cognitive decline (e.g., ventricular enlargement, lesions), strengthening the potentiality of the dog as a model of human aging. The efficacy of using high-field MRI to image dog brain structure was explored by Martín-Vaquero et al. (2011), in which it was found that the 3T MRI provided more consistent and reliable anatomical imaging data than did 7T MRI, contrary to what one might expect given generally superior field strength and image quality at $7 \mathrm{~T}$. In regard to health concerns surrounding MRI with dog subjects, Venn, McBrearty, McKeegan, and Penderis (2014) published findings of post-scan hearing loss, emphasizing the need for hearing protection when imaging dogs in MRI environments.

Though prior research on cognitive process in dogs has been conducted with anesthetized dogs, the cognitive processes of their natural, attentive state are of great comparative interest. The use of anesthesia necessarily impedes attentiveness and alters the state of consciousness, as well as reduces rates of blood flow and respiration. The amalgamation of these reduced biomarkers of stimulus processing leaves much to be desired in the data set, as brain regions or activation patterns involved in cognitive processing may be minimized or lost altogether (Jia, Pustovyy, et al., 2014). In search of valid and viable findings, neuroimaging research with dogs has begun a transition to functional imaging using highly trained dogs that do not require anesthesia for image 
acquisition. In the first published instance of MR images obtained through awake dog imaging, Tóth, Gácsi, Miklósi, Bogner, and Repa (2009) established successful data acquisition with dogs that were trained in a stepwise fashion to remain still and ignore scanner noise. This study consisted of only structural scans, but it was not long after that functional scans were achieved in awake dogs. The movement in canine functional imaging has been pioneered by laboratories at Auburn University, Emory University, and Eotvos Lorand University. Figure 1 documents the timeline of canine fMRI research in awake dogs from these laboratories. Table 1 summarizes the existent literature presenting the number of subjects, tasks, stimuli, and the brain areas activated. Next, we review in a chronological fashion the methods and findings listed in Table 1.

Berns, Brooks, and Spivak (2012) first published research on fMRI data acquisition simple discrimination task in the awake and unrestrained domestic dog. The authors addressed three major challenges in using fMRI technology with dogs: subject motion, which distorts acquired data; use of anesthesia, which eliminates the viability of a cognitive assessment; and immobilization. To target these challenges, the authors developed a set of behavioral and technical methodologies for imaging dogs while they remained motionless, awake, and attentive to a cognitive task. Further, this methodological set was used to assess the reward-prediction error theory of dopamine release in dogs via use of reward signals and attention to activation changes in the ventral striatum. Specifics of this study are presented next.

\section{Proof of Concept}

Two dogs were used as subjects in Berns et al. (2012), one of which had been previously trained in agility. Each dog was incrementally trained, using positive reinforcement, in a mock MRI scanner consisting of a replica of the head coil, scanner bore, and patient table. Additionally, the dogs were exposed to presentations of the scanner noises and sound levels that they would experience in the scanner. The discrimination task was trained by assigning reward

Figure 1. A time-course of canine fMRI research thus far. Recently, this field of research has advanced rapidly and exponentially.

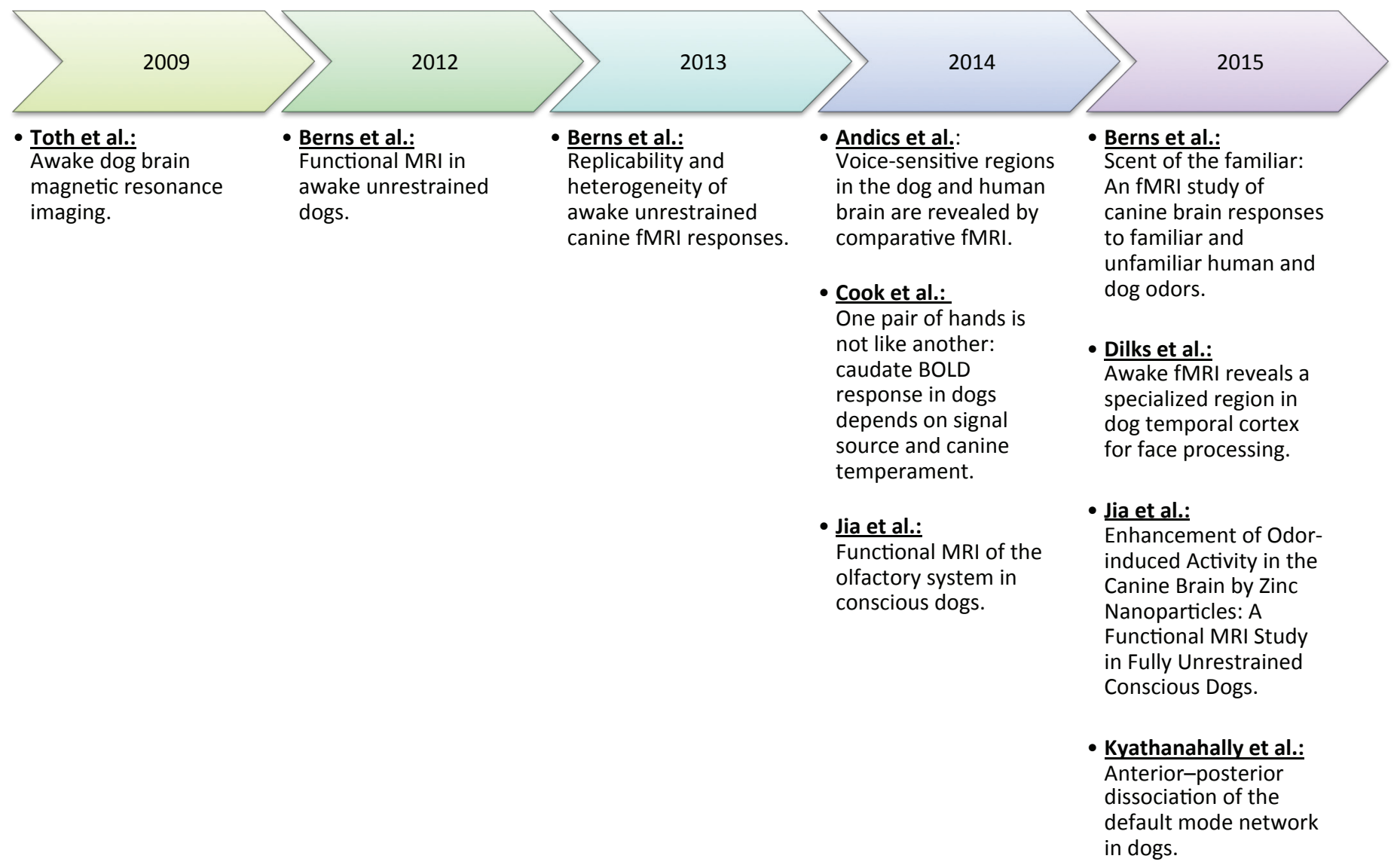


Table 1. Previously Published Awake Canine fMRI Studies.

\begin{tabular}{|c|c|c|c|c|}
\hline Paper & $\begin{array}{l}\text { Subjects } \\
\text { (N) }\end{array}$ & Task(s) & Stimuliº & Area(s) of Activation \\
\hline $\begin{array}{l}\text { Berns, Brooks, \& } \\
\text { Spivak (2012) }\end{array}$ & 2 & Reward expectancy & $\begin{array}{l}\text { (1) Reward hand signal } \\
\text { (2) No-reward hand signal }\end{array}$ & Caudate (right) \\
\hline $\begin{array}{l}\text { Berns, Brooks, \& } \\
\text { Spivak (2013) }\end{array}$ & 13 & Reward expectancy & $\begin{array}{l}\text { (1) Reward hand signal } \\
\text { (2) No-reward hand signal }\end{array}$ & Caudate (left and right) \\
\hline $\begin{array}{l}\text { Cook, Spivak, } \\
\text { and Berns (2014) }\end{array}$ & 12 & Reward expectancy & $\begin{array}{l}\text { (1) Reward hand signal } \\
\text { (2) No-reward hand signal } \\
\text { (a) Familiar human } \\
\text { (b) Unfamiliar human } \\
\text { (c) Computer }\end{array}$ & Caudate (left and right) \\
\hline $\begin{array}{l}\text { Andics, Gácsi, } \\
\text { Faragó, Kis, \& } \\
\text { Miklósi (2014) }\end{array}$ & 11 & Sound processing & $\begin{array}{l}\text { (1) Human nonlinguistic } \\
\text { sounds } \\
\text { (2) Dog sounds } \\
\text { (3) Environment sounds }\end{array}$ & $\begin{array}{l}\text { Both species: Primary Auditory Cortex, Medial } \\
\text { Geniculate Body } \\
\text { Humans: Superior Temporal Sulcus, Inferior Fron- } \\
\text { tal Cortex } \\
\text { Dogs: Perisylvian Regions }\end{array}$ \\
\hline $\begin{array}{l}\text { Jia, Pustovyy, } \\
\text { et al. (2014) }\end{array}$ & 6 & Scent processing & $\begin{array}{l}\text { (1) High concentration odor } \\
\text { (2) Low concentration odor } \\
\text { (3) No odor }\end{array}$ & $\begin{array}{l}\text { Olfactory Bulb**} \\
\text { Piriform Lobes**} \\
\text { Frontal Cortex } \\
\text { Cerebellum }\end{array}$ \\
\hline
\end{tabular}

\begin{tabular}{|c|c|c|c|c|}
\hline $\begin{array}{l}\text { Berns, Brooks, \& } \\
\text { Spivak (2015) }\end{array}$ & 12 & Scent processing & $\begin{array}{l}\text { (1) Human } \\
\text { (2) Dog } \\
\text { (a) Familiar } \\
\text { (b) Unfamiliar }\end{array}$ & $\begin{array}{l}\text { Olfactory Bulb** } \\
\text { Caudate }^{\star \star *}\end{array}$ \\
\hline Dilks et al. (2015) & 8 & Face processing & $\begin{array}{l}\text { (1) Movie clips } \\
\text { (2) Static images } \\
\text { (a) Human faces (MC, SI) } \\
\text { (b) Dog faces (SI) } \\
\text { (c) Objects (MC, SI) } \\
\text { (d) Scenes (MC, SI) } \\
\text { (e) Scrambled objects (MC) } \\
\text { (f) Scrambled faces (SI) }\end{array}$ & Inferior Temporal Cortex (right) \\
\hline $\begin{array}{l}\text { Kyathanahally } \\
\text { et al. (2015) }\end{array}$ & 6 & Resting state & & $\begin{array}{l}\text { Default Mode Network } \\
\text { (anterior cingulate/medial prefrontal areas dissoci- } \\
\text { ated from posterior cingulate) }\end{array}$ \\
\hline Jia et al. (2015) & 14 & Scent processing & $\begin{array}{l}\text { (1) Odorants } \\
\text { (2) Zinc nanoparticles } \\
\text { (3) Gold nanoparticles }\end{array}$ & 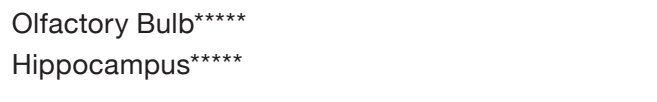 \\
\hline
\end{tabular}

\footnotetext{
${ }^{*}$ greater activation in service dogs

** activation differences by concentration

*** activation for all scents

${ }^{\star \star \star \star}$ greatest activation for familiar human

***** greater activation with zinc nanoparticles

${ }^{\circ}$ Numeric labels indicate primary experiment conditions. Alphabetical labels indicate secondary conditions.
} 
conditions to each of two hand signals given by a handler: a hand held straight up signaled forthcoming presentation of food reward, and two hands held horizontally facing one another signaled no reward.

Once the dogs performed to criteria in the mock scanners, they were moved to true fMRI scanning in a Siemens 3T Trio over a period of six weeks. Initial scanning provided both an assimilation period and an assessment of image acquisition feasibility, followed by a subsequent session to optimize scanning parameters, and finally followed by image acquisition during the randomized instrumental reward task. In this final session, the handler (positioned at the end of the bore) presented 10-second durations of the reward/no-reward task as previously trained.

Analysis of the obtained functional data focused on the head of the caudate in order to target the ventral striatum. The ventral striatum served as the predicted area for activation according to reward-prediction error learning, which anticipates dopamine release and corresponding neural activation of the ventral caudate upon expectation of reward. Reward and no-reward conditions served as the contrast of interest, revealing significant activation differences in the right caudate, though the meaning of the lateralized activation is unclear. These activation differences highlighted a distinct hemodynamic response for reward signal presentations as compared to no-reward signals, thus providing support for the notion that dopamine is released in response to unexpected events that signal future reward and, here specifically, a representation of positive reward prediction in the domestic dog.

\section{Replication of the Reward/No-Reward Task}

Berns, Brooks, and Spivak (2013) followed their initial 2012 study of fMRI with dogs with an assessment of the replicability of their methodology. Further, the authors sought to potentially reduce signal variability of caudate responses to the instrumental reward task with additional experimental improvements. In this replication, $13 \mathrm{dogs}$ of various training background (e.g., service, agility, basic obedience) completed positive-reinforcement training on the mock scanners (this time, with a mock knee coil instead of head coil) and the reward/no-reward task.

In this expanded subject set, $62 \%$ of dogs showed significant differential positive activation in the caudate for reward signals. These findings were consistent with Berns et al. (2012); however, substantial signal variability was found across subjects for overall caudate activation. Berns et al. (2013) discuss several potential reasons for this variability between subjects, including greater human attachment in service and therapy dogs, the inherent noise of imaging data, the difficult balance between imaging repetition and efficacy of the task, mislocation of regions of interest, and individual motivational differences. Interestingly, the authors note that when the dog fMRI data collected from the instrumental reward task is compared to that of humans, it may indeed be less variable than human caudate activity. Overall, this replication of awake, unrestrained fMRI with dogs supported the efficacy of reliable training in demonstrating differential activations in the dog brain. Further, the results of this study and Berns et al. (2012) provide support for the possibility of dog models of human cognitive function.

\section{Temperament and Stimulus Source}

To further expand on their developments in dog fMRI, Cook, Spivak, and Berns (2014) modified their reward/ no-reward task to assess activation differences driven both by subject temperament and stimulus source. The same dogs as used in Berns et al. (2013) were employed in this study, and all were evaluated for 14 factors of temperament (e.g., attachment, trainability, Hsu \& Serpell, 2003) using the owner self-report Canine Behavioral Assessment and Research Questionnaire (C-BARQ). Stimulus sources were divided equally among reward/no-reward hand signal presentations given by a familiar person or an unfamiliar person, as well as digitized hand signal displays presented on a projection screen. Analyses revealed that across the subject set, the caudate was differentially active by condition, indicating further support for the implication of the ventral striatum in reward anticipation. Further, activations revealed that the dogs could generalize the meaning of the hand signals across stimulus sources. When C-BARQ temperament factors, particularly aggressivity, were taken into account, activation differences were found according to a stimulus source of familiar human versus unfamiliar human or projection by computer. That is, dogs with lower aggressivity levels showed greater activation for reward signals given by the familiar person than by the unfamiliar person or computer. Alternatively, dogs with higher aggressivity levels showed greater activation for reward signals given by an unfamiliar person or computer. Cook et al. (2014) note that, because the striatal response is dependent upon arousal and stimulus salience, higher aggressiveness correlates to higher salience for the novel situations of unfamiliar person and computer, and that lower aggressiveness correlates to lower anxiety and higher salience with a familiar person. In their conclusion, the authors stress the possibility of differences across dogs in their reactions to various contexts, and emphasize the need for consideration of this possibility when making claims from dog studies without temperament testing. 
In all, these initial studies of functional imaging with dogs provided strong support for the opportunities presented by the merger of canine cognition with fMRI technology. The establishment of successful training and imaging techniques allows for the expansion of this research to involve more specific regions of interest along with a greater range of subjects and ontogenic histories. Notably, success with visually based experiments provided an interesting opportunity to investigate processing in other sensory modalities.

\section{Audition}

Andics et al. (2014) have also used positive-reinforcement training to conduct fMRI studies with awake and unrestrained dogs. Here, the authors conducted comparative research into the function and location of voice-sensitive brain regions in dogs and humans. Because humans and dogs have long shared a natural environment, Andics et al. (2014) questioned how voice-sensitive regions in both populations would respond to conspecifics and heterospecifics, and whether they would show similar processing of emotional cues in these signals. Eleven dogs and 22 humans participated in scans during which an identical set of auditory stimuli was presented. This stimulus set consisted of human (e.g., laugh, cough, yawn) and dog vocalizations (e.g., growl, pant, bark) ranging in emotional valence along with environmental sounds and silence. The silence condition was used to functionally assign the auditory region of interest by contrasting the fMRI response to silence against the response during sound presentation.

Cortical sound sensitivities were revealed in the perisylvian regions for the dogs and the superior temporal sulcus and inferior frontal cortex for humans. Both species showed sensitivity in the medial geniculate body. In the dog brain, subregions were identified that activated maximally for dog vocalizations as well as to human vocalizations and environmental sounds. On the contrary, nearly all human auditory regions of activation were maximal for human vocalizations, although the medial geniculate body showed a maximal activation for dog vocalizations.

\section{Olfaction}

Jia, Pustovyy, et al. (2014) utilized positive-reinforcement training and fMRI with awake and unrestrained dogs to investigate olfactory processing and the effects of anesthesia on the quality of neural data. The authors note that there is a large body of literature pertaining to both the cellular and behavioral correlates of olfaction in dogs, but little research has been done on the cognitive processes that underlie olfaction. Thus, their study aimed to serve as a comparison of the neural response in the brain to varying odor concentrations in awake versus anesthetized dogs. Six dogs served as the subjects for this study, and a specialized computer-controlled odorant delivery system was designed with MR safety restrictions and parameters in mind (e.g., elimination of ferromagnetic objects in the scanner room, motion control). This delivery system was used to precisely present 10 -second periods of odorant (ethyl butyrate, eugenol, \& carvone mixture) to dogs across five randomized blocks. Further, for the first time in canine fMRI research, these authors used a single external infrared camera to track dog head motion and retrospectively correct for motionrelated artifacts in the data, especially faster and jerky movements which cannot be captured by the poor temporal resolution available to image-based rigid body registration methods which are commonly used in fMRI analysis.

Both awake and anesthetized dogs demonstrated strong activation in the olfactory bulb and bilateral piriform lobes upon presentation of both high and low concentrations of odor. However, the intensity of activations, as well as their spatial extent, was mediated by concentration, with larger activations for higher odor concentrations. Awake dogs exhibited activations in areas including the medial, superior, and orbital frontal cortices and the cerebellum, all of which are tied to cognitive processes, whereas anesthetized dogs did not. Given the findings, the authors concluded that anesthesia degrades processing of odors and that the use of fMRI can and will provide a useful investigation into the neural substrates of the olfactory system.

Recent findings by Jia et al. (2015) expanded on this work and revealed olfactory enhancement with the addition of zinc nanoparticles to odorant presentations. Using conditions of pure odorants, odorants plus zinc nanoparticles, and gold nanoparticles, as well as zinc nanoparticles alone in water vapor, Jia et al. (2015) hypothesized that zinc nanoparticles, previously implicated in enhancement of odor response in vitro, would lead to greater activity in the brain regions for olfactory processing that were revealed in Jia, Pustovyy, et al. (2014). Indeed, activations in the olfactory bulb and hippocampus were greater in awake dogs exposed to odorants with zinc nanoparticles compared to pure odorants, pure zinc nanoparticles, and odorants with gold nanoparticles. Acknowledging the need for confirmation of increased odor sensitivity via behavioral tests, Jia et al. (2015) highlight the possible utility of zinc for enhancing the abilities of working odor detection dogs.

Berns, Brooks, and Spivak (2015) sought to investigate the canine perceptual experience of socially related stimuli via the processing odors of familiar and unfamiliar people and dogs. In order to investigate the driving social relationship between a human and dog, the authors again utilized 
the dopamine theory of reward-error prediction, hypothesizing that if the relationship between a dog and its most familiar person includes reward expectancy, then caudate activation will be greater when the scent of that person is being processed, as opposed to another person or a dog. The same dogs that were used in their prior research (Berns et al., 2012; Berns et al., 2013; Cook et al., 2014) were enlisted for this study. Additional training was needed to acclimatize the dogs to smelling odors on a cotton swab while withholding approach. For presentation of swabs during scanning sessions, odors for the familiar and unfamiliar human were obtained from the armpits, and odors for the familiar dog, unfamiliar dog, and the dog's own self were obtained from the perineal-genital areas. In order to maintain compliance and motivation, the dogs were presented with interspersed reward trials during odor-presentation runs.

Analyses of the obtained imaging data focused on two regions of interest: the olfactory bulb and the caudate nucleus. The olfactory bulb was generally significantly activated by the task and this activation was non-differential across all five odor types. However, the caudate nucleus showed differential activation according to odor type. For all dogs, the caudate was maximally activated for the odor of a familiar person, suggesting that a positive reward association is in place for the scent of a familiar human, even in their physical absence. Interestingly, service dogs once again stood out with greater overall caudate activation as compared to dogs with other histories.

Collectively, the studies conducted by Jia, Pustovyy, et al. (2014) and Jia et al. (2015) and Berns et al. (2015) provide evidence for the efficacy of olfactory neuroimaging with dogs. The olfactory bulb has been consistently implicated in the processing of odors, and the use of anesthesia and the intensity of odors have been directly tied to olfactory processing. Additionally, reward-based processing of odorants was supported by activations in the caudate nucleus. Given the findings of these studies, there is clear evidence that fMRI can be utilized for future research to systematically explore olfactory processing in dogs.

\section{Face Processing}

In the first published fMRI investigation of face processing in awake dogs, Dilks et al. (2015) presented eight fMRIexperienced canine subjects (Cook et al., 2014) with movie clips and static images. The dogs viewed movie clips of human faces, scenes, objects, and scrambled objects, each for three seconds. In the static images condition, the dogs were presented with black and white images of human faces, dog faces, objects, scenes, and scrambled faces, each for 600 milliseconds. Imaging data was analyzed for six of the dogs, and movie clip contrasts localized dog and human face processing to the inferior temporal cortex in the right hemisphere. The data also revealed significant category effect for static images when face images were compared with objects and scenes. Because the response profile did not map onto the dog visual cortex, low-level feature processing is unlikely to account for the activation patterns seen in the temporal lobe. Rather, Dilks et al. (2015) conclude that the activations represent the first evidence of a face-processing region in dogs.

\section{Resting State}

The methodology of resting state fMRI has gained traction in the past decade because of distinct advantages it offers in terms of experimental design. Foremost, it does not require the subjects to perform any task, and hence is less stressful to subjects in human patient populations. Next, task-based activation studies have to be carefully designed so that any differences in responses may not be attributable to differences in task performance metrics (such as accuracy and reaction time). These measures may not be always possible to achieve. No such requirements are placed in resting state studies. Finally, analysis of task-based activation studies are primarily model driven (although data-driven methods also exist, it is difficult to interpret all time-locked evoked responses obtained from them), and this poses a challenge because one would have to explicitly model all sources of variance in measured data. However, in resting state studies, one could simply correlate experimentally measured time series from different brain regions (or perform an independent component analysis) to uncover underlying brain networks which are coevolving in time. These advantages of resting state studies in the human context are even more applicable in the context of awake dog imaging, as it is harder to make dogs perform a task (active or passive) and assure compliance and uniform performance.

Kyathanahally et al. (2015) used resting state fMRI to identify whether the default mode network (DMN), found reliably in humans (Buckner, Andrews-Hanna, \& Schacter, 2008) and monkeys (Mantini et al., 2011) but much less frequently in rodents (Becerra, Pendse, Chang, Bishop, \& Borsook, 2011; Upadhyay et al., 2011), exists in the domestic dog. Resting state fMRI is conducted with subjects that do not perform any cognitive tasks, but rather lie still with eyes open and relax. In humans, the core part of the DMN is active during rest and consists of two connected subnetworks - the posterior part consisting of the posterior cingulate cortex (PCC) and inferior parietal cortical areas, as well as an anterior part consisting of medial frontal structures. (Note that we are referring to the core part of the DMN and 
not the extended DMN, which also consists of lateral and medial temporal cortices. Please see Buckner et al., 2008, for details.) This network has been implicated in cognition and self-referential processing, and it has been found reliably in human resting state fMRI investigations. Additionally, this network's activity is depressed when a patient is under anesthesia (Greicius et al., 2008). Most importantly, though, the long-range connectivity between the anterior and posterior parts of the DMN is lacking in very young children and seems to develop with age in humans (Fair et al., 2009). The establishment of long-range connectivity between anterior and posterior parts of the DMN is thought to facilitate large-scale information integration required for higher cognitive processes. Further, in humans, connectivity magnitude and associated network structure for various resting state networks, specifically the DMN, have been shown to be more informative in predicting behavior as well as traits compared to activation alone (Cole, Yarkoni, Repovš, Anticevic, \& Braver, 2012; Jia, Hu, \& Deshpande, 2014). Therefore, investigation of resting state networks in awake dogs is a promising area of research.

To assess the presence of a DMN in dogs and to understand the effects of anesthesia on its activation, Kyathanahally et al. (2015) scanned six dog subjects in both awake and anesthetized states. Seed-based and independent component analyses (ICA) were used and identified dissociation between the anterior and posterior regions of the DMN. Further, while this dissociation was seen for both awake and anesthetized dogs, the degree of dissociation was higher in anesthetized dogs in keeping with prior human results that anesthesia modulated the structure of resting state networks such as the DMN (Deshpande, Kerssens, Sebel, \& Hu, 2010). In all, this investigation into resting state fMRI with dogs revealed comparative differences in the traits of the DMN between humans/monkeys and dogs, namely localized anterior and posterior subnetworks in dogs and a connected DMN in humans. The findings suggest differences in cognitive processing that are perhaps due to evolutionary differences.

In summary, the research and findings discussed herein are representative of the current excitement and expansion of canine cognitive research into functional imaging. As interest and conceptual foundations in this area continue to grow, the cognitive processes and behavior of the domestic dog may be better linked to develop a comprehensive understanding of man's best friend. Further, such linking of cognition and behavior will allow for more informed comparisons to be made across species, as well as allow for greater understanding of the environment effects of domestication into the human social world. Though this area of research offers much promise, there are many challenges left to be addressed, both in respect to training and imaging methodologies and conceptual issues of cognitive investigation. In the following sections, we review those challenges most pertinent to future canine neuroimaging studies.

\section{Methodological Issues and Solutions}

The ability to obtain fMRI data on awake and unrestrained dogs provides expansive opportunity for research, but the advancement of this body of research does not come without significant methodological requirements and considerations. Here, we outline a variety of requisite considerations for safe scanning of dogs and provide solutions for each (Figure 2). We explore safety concerns, potential stressors for dog subjects, suitable scan parameters, desired experimental rigor, and the generalizability of imaging results to the greater dog population.

\section{Safety}

An important methodological concern of fMRI with dogs is the well-being and safety of individual canine subjects. Due to the extremely high sound pressure levels (SPLs) found in the MR environment, noise-induced discomfort and hearing loss are a concern for MR experiments.

Figure 2. A representation showing a flow of the methodological concerns to be addressed in design and execution of fMRI experiments with dogs.

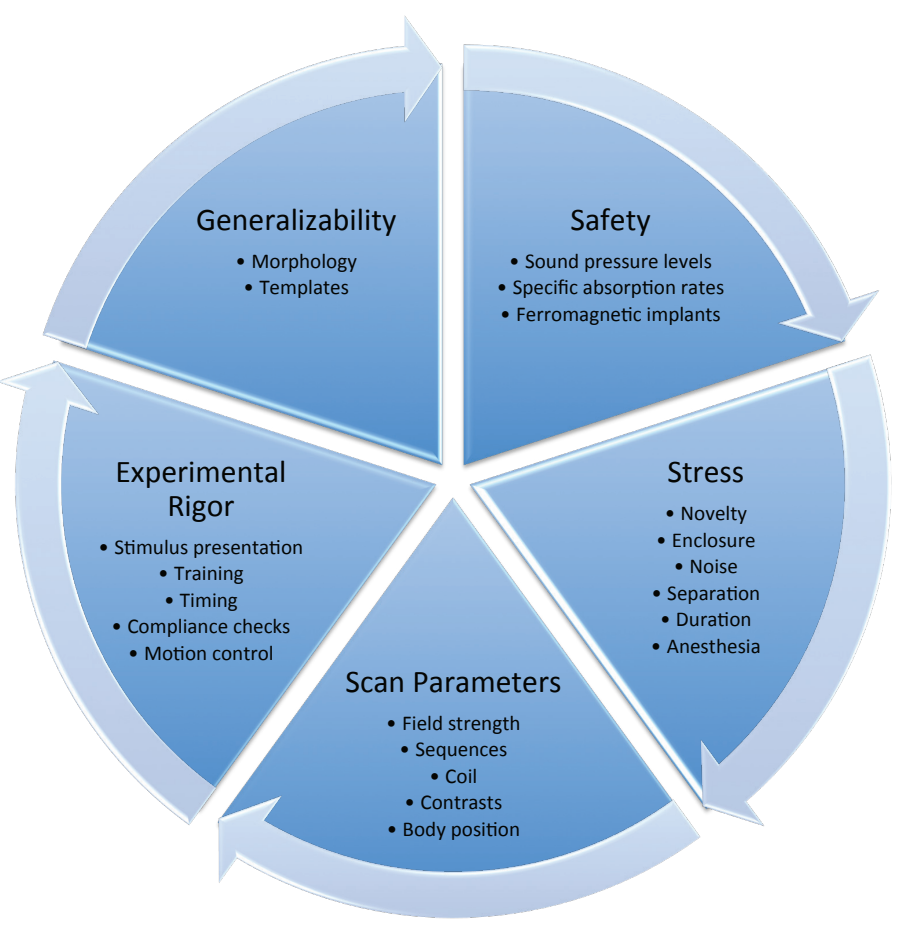


Sound levels in an MR suite range between 65 and $95 \mathrm{~dB}$, with peaks from 120 to $131 \mathrm{~dB}$, and these levels have been shown to result in significant short-term hearing loss in dogs (Venn et al., 2014). Physiological effects of sound stress include elevations in heart rate and blood pressure, as well as changes in metabolism. Additionally, the noise experienced in MRI may cause inner ear pain, distress, and inhibited communication abilities in dogs (Lauer, El-Sharkawy, Kraitchman, \& Edelstein, 2012). Though long-term hearing loss due to scanner exposure has not been investigated in dogs (for long-term effects of cochlear damage in mice, see Kujawa \& Liberman, 2009), such long-term effects could prove catastrophic to the safety and trainability of dogs, especially those working in search and rescue, bomb detection, and police work, as they are often physically separated from their handlers and require attention to vocal cues at a distance.

When designing experiments for canine fMRI, the most desirable solution to the problem of scanner noise is the use of sound-attenuating earmuffs in combination with careful selection of scan parameters. Earmuffs such as "Mutt Muffs" can provide upwards of $28 \mathrm{~dB}$ of sound reduction when fitted properly to each dog (www.safeandsoundpets. com) and have been successfully used in past experiments (Cook et al., 2014). For further noise reduction where experimental demands allow, the use of a "whisper" mode and/or alterations in scanning parameters can attenuate sound pressure levels during scanning (Baker, 2013).

In addition to noise levels, safety concerns arise regarding the specific absorption rates (SAR) of radio frequencies for dogs. When RF energy in the scanner is absorbed by the body, tissues may rise in temperature. Tissue heating is almost always negligible; however, in order to eliminate the risk of thermal injury, SAR levels should be measured throughout MR scans (Smith, 2010). A variety of scan parameters influence SAR levels, including frequency, TR, coil selection, and the orientation of the body. Further, RF absorption by the body is determined by exposure duration, the thermoregulatory system, and health conditions (Shellock \& Crues, 2004). In the case of human patients, the Food and Drug Administration (FDA) has established guidelines for the maximum allowable radio frequency absorption by tissue, defined as 4 watts per kilogram for a period of up to 15 minutes, or 3 watts per kilogram over the head for a period up to 10 minutes. Unfortunately, no such guidelines exist for nonhuman subjects, and this makes it the responsibility of researchers using dogs in MRI experiments to assess the SAR levels experienced by subjects. Until such research has been conducted, it seems best to adhere to the FDA standards for humans when working with dogs inside the scanner (Berns et al., 2013). Additionally, Berns et al. (2013) note that a reduction in flip angle may prevent rises in SAR levels, as do shorter scans. Given that SAR values generally increase with body weight, adhering to human SAR levels may be enough to protect the dogs, assuming that humans in general weigh more than domestic dogs. Nevertheless, it is a good idea to weigh the dogs and enter their body weight while running MR sequences so that the scanner can get a realistic estimate of SAR levels for individual dogs.

A large portion of the domestic dog population that may be ideal for study in the MRI environment is without recorded life history. That is, in many cases, there is some period of a dog's life history that cannot be accounted for in terms of potential safety hazards (e.g., metallic objects in the body) or medical ailments and/or procedures. Because of this gap in life history, safety precautions must be taken before placing a dog in the MR environment. Of key concern is the potential for ferromagnetic objects that have been implanted in or ingested by a dog (Smith, 2010). The presence of conductive materials within the body may lead to excessive heating and third-degree burns (Shellock \& Crues, 2004). Due to this potential hazard, it is imperative that each dog be screened for the presence of metallic objects in the body before being enlisted in an experiment and entering the scanner environment. Shellock and Crues (2004) note that while an object may be demonstrated as safe under a given set of MRI conditions, the same object may be unsafe in other conditions, especially those using stronger fields, greater radio frequencies, and different RF coils. This must be taken into consideration when assessing individual dogs for new scan conditions or replications in new scanner environments. Sensitive hand-held metal detectors custom-built for MR prescreening must be employed prior to the dog's entering the scanner room in order to make sure that the dog does not have any ferromagnetic material inside its body.

\section{Stress}

Alongside the importance of safety within the scanner is the need to eliminate undue stress to the animal being investigated. Undue stress may prevent generalizability of the data. Stress may be defined as something that challenges the homeostasis within an individual or that places demands on the individual for which they do not have adequate resources. Such a stressor leads to physiological and behavioral responses that engage and mobilize the animal for action. Short-term stressors (such as the scanner environment) lead to increases in vigilance, alarm, and orientation, as well as physiologic responses such as tachycardia, metabolic changes, and increased respiration 
(Morgan \& Tromborg, 2007). King et al. (2005) note that increased respiration and heart rate, as well as behavioral changes such as head motion, may alter the BOLD signal in such a way that activation changes in the brain may partially be attributed to noise rather than just the manipulation of an experimental variable.

In experiments aimed at acquiring imaging data from dogs, sources of stress may include noise, environmental novelty, enclosed spaces, restriction of movement, separation from the owner/handler, and long durations of assessment. Currently, the different techniques employed by dog neuroimaging laboratories inherently address stress reduction in training, as they lead to willful compliance. Methodologies using progression from mock to true scanning (e.g., Berns et al., 2012; Jia, Pustovyy, et al., 2014) gradually introduce dogs to the space constraints, noise levels, and time requirements of the scanning environment. A similar note can be made of the gradual introduction for dogs participating as observers in the model-rival method (Andics et al., 2014). Gradual exposure not only allows for reduced risk of stress in an overwhelming environment, but also gives trainers an opportunity to identify and eliminate stress signals emitted by a dog at any point in training. Acclimating dogs to the aforementioned sources of stress during training is essential to successful scanning, and improvements in training techniques aimed at further reducing sources of stress will be important for future research. In addition, stressors not accounted for or eliminated by training methodologies may still be reduced by thoughtful selection of experimental parameters, and such parameter manipulations should be explored.

Auditory experiments in fMRI pose a unique challenge due to the high sound pressure levels within the scanner room. In order to optimize the amount of auditory stimuli that can be heard and processed, a sparse temporal sampling (STS) procedure may be used, as in Bach et al. (2013) and Andics et al. (2014). This sort of imaging paradigm allows for periods of scanner silence during which the auditory stimuli of interest may be presented without interference. Because the BOLD response lags in time behind the neural response, acquiring imaging data shortly after, but not during, auditory stimulus presentation allows capture of the stimulus-evoked hemodynamic response function. Bach and colleagues (2013) implemented this procedure and compared it with a standard scanning procedure without intermittent silent periods. Though the dogs were anesthetized and some degree of signal attenuation could be expected, the authors found reliable activation of auditory areas for all of the dogs. Of interest to the use of STS, conditions during which auditory stimuli were presented during silent period resulted in significantly higher activation levels than those presented during continuous scanning, supporting past evidence that STS procedures may increase signal strength by $21 \%$. When using awake dogs for auditory fMRI experiments, the use of STS procedures is an ideal way to ensure optimal BOLD signal and may be easily introduced into positive-reinforcement behavioral training.

\section{Scanning Parameters}

As mentioned in relation to noise and stress reduction, careful selection of the imaging sequence and scanning parameters is an essential component of canine fMRI methodology. While we do not attempt to explain all technological aspects here, interested readers may find the cited articles useful. Goals of parameter selection are aimed at increasing efficiency in data collection and in subsequent processing of obtained data. Parameters of consideration include the choice of (a) field strength, (b) imaging sequence and its parameters, (c) RF coil, (d) signal contrast, and (e) body position. First, in some cases, the experimenter may have the option of scanning in a 3T or 7T scanner. MartínVaquero et al. (2011) sought to parse out differences in image quality between the two field strengths using anesthetized dogs. Contrary to what might be expected, only eight of 32 anatomical structures had better image quality in the 7T scanner as compared to the 3T. Most structures (19/32) were of comparable image quality for both scanners, and five were better at 3T field strength. Specifically, MartínVaquero and colleagues found that when performing highresolution scans, the noise due to magnetic susceptibility and chemical shift were much more apparent in the 7T scanner, and thus suggest using a 3T scanner for these sequences. However, this contradicts a large body of evidence that suggests that increasing field strengths offers substantial benefits in terms of SNR (Duyn, 2012). Unlike the 3T, getting fMRI data of good quality from $7 \mathrm{~T}$ requires the choice of proper sequence parameters (which change with field strength) and use of higher order shimming. Therefore, if done right, higher field strengths such as the $7 \mathrm{~T}$ could potentially offer increased SNR and smaller voxels, which are crucial in canine imaging since brain structures in dogs are relatively smaller than in humans. Such advantages of ultra-high field have already been demonstrated for imaging relatively smaller structures in the human brain (Denison, Vu, Yacoub, Feinberg, \& Silver, 2014; Satpute et al., 2013; Suthana et al., 2015) as well as other smaller mammals such as rodents (Schafer, Kida, Xu, Rothman, \& Hyder, 2006).

Second, in regard to choice of sequence, Chen et al. (2012) found that in MR imaging of awake monkeys, the signal-to-noise ratio (SNR) was reduced by using segmented 
echo-planar imaging (EPI) in exchange for single-shot EPI, and also by optimizing echo time. Recent innovations such as the multiband EPI (Feinberg et al., 2010) will allow us to choose a shorter TR for the same voxel size compared to regular EPI. Also, the use of parallel imaging in sequences may not only reduce scan time and acoustic noise, but also be beneficial for mitigating artifacts due to off-resonance effects (Golay, de Zwart, Ho, \& Sitoh, 2004). Further, zoomed resolution approaches may be employed for obtaining higher spatial resolution from specific structures (Yacoub, Harel, \& Uğurbil, 2008). For performing dog fMRI in 3 T scanners, which are widely available, the choice of scan parameters depends on the scientific question being investigated. The available SNR could be traded for either spatial or temporal resolution. Therefore, if the scientific investigation surrounds smaller structures such as the nucleus accumbens or other nuclei in the brain stem, then it may be a good idea to have smaller voxel size and a longer TR. However, if one is interested in temporal properties of the signal in relatively larger regions of the cortex, employing a shorter TR and relatively larger voxel size may be preferable. In general, since the size of the dog brain is smaller than that of the human brain (Roth \& Dicke, 2005), scanning sequences must strive for achieving voxel sizes that are smaller than that typically used in human scans. Briefly returning to stress and safety concerns, the experimenter must also make note of the effects of timing and slice selection on noise and scan duration and select accordingly in order to create optimal experimental conditions.

Third, as noted before, different types of coils (e.g., head, neck, human knee, flex) have been used by different investigators. It is difficult to compare the SNRs obtained from these coils since corresponding raw data is not available in the public domain. Nevertheless, an important factor to bear in mind irrespective of the coil used is that one needs to ensure that the coil is close to the dog's brain. Standard quality control procedures must be employed to assess whether the signal obtained from a coil is of acceptable quality. However, given the wide range of options available (such as surface coils, linear volume coils, birdcages, phased arrays), further studies are required to determine what type of coil is necessary and sufficient for performing routine fMRI in awake dogs. Specifically, efforts must be made for developing size- and shape-matched head coils for imaging the canine brain for achieving both high sensitivity and specificity. If successful, the higher performance obtained from a custom-built coil may aid in obtaining much higher temporal and spatial resolution, as well as much larger functional contrast-to-noise ratio. Shorter measurement times could help to avoid image deterioration by motion artifacts, for instance physiological motion artifacts due to respiration and heart rate within the skull. The performance of such a custom-built coil could then be compared with human coils that have been adapted for canine imaging so far in order to determine whether the investments required for building custom coils in terms of capital and technical expertise are indeed justified.

Fourth, various options are available for imaging function in the brain using MRI. These include the widely used BOLD signal contrast, perfusion-based methods such as arterial spin labeling (ASL; Petcharunpaisan, Ramalho, \& Castillo, 2010), and Vascular space occupancy (VASO; Lu \& van Zijl., 2012). However, the use of signal contrast mechanisms other than BOLD in animal research has been primarily driven by pharmacological fMRI in preclinical studies (Nasrallah, Lee, \& Chuang, 2012). Unless a study is using a dog model of human illness and testing pharmacological effects of different drugs, the BOLD contrast provides the right ingredients in terms of sensitivity required for probing normal cognition in the canine brain. Finally, all canine fMRI studies published so far have used dogs in the prone position, which seems like a natural choice. Further, the coil geometry also lends itself to this position very well. We do not see a reason to image dogs in other positions such as supine, unless specifically required for an application.

\section{Experimental Rigor}

Given that canine fMRI is a nascent field, initial reports were more interested in demonstrating the proof of concept rather than follow experimental rigor that is customary in human fMRI studies. We can observe the following shortcomings: (a) lack of controlled delivery of stimuli such that they are devoid of subjectivity and timing error (e.g., Berns et al., 2012), which can be detrimental in general linear modeling of the BOLD signal, (b) temporal discontinuity between trials (e.g.. Berns et al., 2013), which can invalidate assumptions made during activation analyses, (c) small sample sizes resulting in smaller effect sizes, (d) lack of compliance measurements, and (e) inadequate attention toward motion artifacts. Given that the field has moved beyond the proof-of-concept stage, we suggest that future studies make every attempt to employ best practices used in human fMRI studies. For example: (a) given that controlled delivery of olfactory stimuli by a custom-built device was demonstrated by Jia, Pustovyy, et al. (2014) and likewise with auditory stimuli by Andics et al. (2014), similar approaches could be used while presenting stimuli of other modalities as is routinely done in human fMRI research; (b) training dogs to remain in the scanner for a longer period of time (>1-2 minutes, as in Jia, Pustovyy, et al., 2014, and 
Andics et al., 2014). would naturally obviate the necessity to have the dog pull out of the coil after every trial (or two) or move between trials, which introduces temporal discontinuities in the signal and necessitates that data be discarded; (c) recruiting larger numbers of dogs and/or obtaining more runs from available dogs coupled with spatial normalization techniques (discussed next) would allow group inferences with robust statistics rather than qualitative inferences in individual dogs; (d) if dogs are presented visual stimuli, manual checking of compliance by other humans or automatic compliance measurement via eye-tracking is desirable.

In regard to motion control, there is increasing awareness in the neuroimaging community about the detrimental effects of head motion on fMRI data quality (Power et al., 2014). Canine fMRI studies have taken comfort in the fact that the motion parameters obtained from rigid body registration (i.e., three translations and three rotations) can be inspected to choose only data not corrupted by motion (e.g., Berns et al., 2013). However, this approach does not take into account the facts that: (a) spin history effects and throughplane motion are not modeled in rigid-body registration or by censoring only affected TRs, (b) the frame-wise displacement of different voxels in the brain are different from each other, and (c) rapid motion that occurs between TRs can affect data quality in ways that cannot be restored by rigidbody registration or even censoring. Some of these issues were partially addressed by Jia, Pustovyy, et al. (2014) and Kyathanahally et al. (2015) by employing a single external infrared camera to record dogs' head motion with high temporal resolution (order of milliseconds) and spatial precision (order of micrometers) and then correcting for those effects post hoc. However, in an ideal scenario, we suggest employing prospective motion correction by either employing an external camera (Todd, Josephs, Callaghan, Lutti, \& Weiskopf, 2015; Maclaren et al., 2012) or using imagebased tracking (as in 3D PACE; Thesen, Heid, Mueller, \& Schad, 2000).

\section{Generalizability}

Across breeds, the domestic dog demonstrates significant variability in brain morphology (Roberts, McGreevy, \& Valenzuela, 2010) and therefore presents a challenge when attempting to spatially normalize images into a stereotactic space and generalize findings from fMRI experiments with dogs. In order to encourage spatial normalization across experiments for imaging data obtained from canine fMRI, Datta et al. (2012) used 15 mesocephalic dogs to create a 3T template. However, they note the influence of the mesocephalic characteristics of the subjects and suggest that differences in encephalization may lead to differences in cortical folding that preclude simple transformations, rendering the normalized template suitable only for dogs similar in skull and brain structure to those used in development of the template. Such concern is evidenced by brain structure analyses conducted by Roberts et al. (2010), in which it was found that brachycephalic dogs possess skulls with comparatively rotated cerebral hemispheres, pitched brain angle at the anterior pole, and repositioning of the olfactory lobe. To combat the exclusivity of normalized images due to such differences, Bach et al. (2013) suggest a wider scope of canine structural imaging to include a variety of skull shapes. Along those lines, they suggest the development of a database similar to what is seen in BrainMap for human data (Laird, Lancaster, \& Fox, 2005), where imaging data from a multitude of canine fMRI studies may be accumulated, analyzed, and normalized. In the meantime, thought should be given to the viability of using a normalized template with a given population of dogs. For those not matching optimally to the template, it will be best to use within-group spatial normalization as seen in Jia, Pustovyy, et al. (2014).

\section{Conceptual Issues and Solutions}

Once the canine researcher has addressed the methodological challenges described above, another set of challenges related to conceptual foundations must be addressed. Of utmost importance is the knowledge of what is already known about the structure and function of the domestic dog brain. This knowledge provides the framework with which to build viable hypotheses and draw conclusions from sometimes convoluted activation data. When developing these hypotheses and conclusions, it is also important to use a theoretical background and logical arguments in order to parse out the many possibilities for structure-function relationships. Steps to develop this conceptual framework will now be discussed.

Of great benefit to both fMRI methodology and comparative cognition research is the shared natural environment of dogs and humans. Miklósi and Topál (2013) discuss the exploration of "human-like" and "infant-like" functional traits in dogs and emphasize the importance of careful control in experiments aimed at identifying cognitive mechanisms. When it comes to domestic dogs, criteria of taskdemand and environmental similarity have already been met and are easily accounted for when designing a task. This is because the natural environment of dogs is the human environment, rather than the environment of their distant relatives and ancestors. Because dogs are encultured in human society, there are far fewer methodological concerns 
regarding environmental generalizability when it comes to interaction with experimenters, presentation of commands and/or rewards by humans, stimuli found in the human environment, and ambient aspects such as sound and lighting. Here, these aspects of generalizability can be assumed so long as the experimenter takes strides to equate exposure and task difficulty for humans and dogs.

The crux of in-scanner validity may be the mode of stimulus presentation. As with most laboratory experiments, the ability to replicate real-life scenarios in the scanner is limited, as visual stimuli are typically presented via projector screen, auditory stimuli via speaker or earphone, and olfactory stimuli via localized sampling. Because of the constrained nature of such presentations, skeptics may rightly question whether activations are representative of what would occur in a natural situation. Evidence from prior canine fMRI studies would suggest that imaging data from experimental analogs are at least indicative, if not wholly representative, of real-world scenarios (e.g., auditory samples, Andics et al. 2014; olfactory samples, Berns et al. 2015). However, findings from other research keep certainty of generalizability at bay. For example, Snow et al. (2011) found that in humans, patterns of activation differed between presentation of 3D objects and 2D images, highlighting that $3 \mathrm{D}$ objects offer more information about a stimulus than a 2D image of the same stimulus, and may even provide motivation for attention due to the possibility of interaction. Given the possibility of generalizability risks and the lack of literature investigation regarding this risk in dogs, researchers should attempt to eliminate these concerns by providing naturalistic stimuli whenever possible. As noted, this issue of stimulus generalizability between stimuli in the testing environment and the real world (or 2D vs. 3D stimuli) is not unique to the MR scanner environment (e.g., Spetch, 2010). However, steps should be taken to minimize the discrepancy between stimuli and their real-life counterparts as well as acknowledge this remaining discrepancy when analyzing and discussing imaging data.

By its nature, fMRI requires multiple instances of neural activations and corresponding BOLD responses to create a clear localization of function in the brain. Due to this repetitive nature, attention and habituation become concerns as number of presentations and time in the scanner increase. These concerns are exacerbated in dogs, as there is not a good means of communicating the need for attention, nor is there a desirable method for tracking decreasing attention span or habituation while behavioral responses are being withheld. Snow et al. (2011) found that when presenting human participants with repeating instances of a single 2D image, functional data showed robust repetition effects and degradation of signal. It is desirable to be cautious of such effects in canine data, as similar repetition effects have been found in non-human primates (Miller, Li, \& Desimone, 1991). Further, continued attention to any stimuli is a concern when requiring dogs to remain still for extended periods of time. Cook et al. (2014) anticipated attentional changes with increased scan duration when presenting dogs with familiar human, unfamiliar human, and computerized image presentations of reward signals. To combat deficits in attention, the researchers arranged the experimental conditions such that the signals presented later in scanning would be more stimulating and motivational (in this case, presentations of a familiar human). The disadvantage of such an approach would be introduction of order effects.

In any fMRI experiment, there exists the possibility of overzealous or inaccurate connections being made between structure and function. This becomes especially true when attempting to separate active and passive processing in nonhuman animals, as we cannot be sure what sort of cognitive process they are engaging in without concurrent behavioral measures. Whereas human participants may exhibit a given cognitive process behaviorally during a scan (e.g., via a response box) or report on strategies after scanning, we do not have the luxury of obtaining such information from dog subjects. The importance of distinguishing between active and passive processing is great, as human research has shown differential activations between the two cognitive processes (e.g., O’Craven, Rosen, Kwong, Treisman, \& Savoy, 1997). As a first measure to preventing inaccurate designation of cognitive process to activation, researchers can ensure that dogs have had extensive out-of-scanner training on a behavioral measure for the cognitive task in question. For example, in the reward signal research previously discussed (e.g., Cook et al., 2014), dogs were extensively exposed to the experimental conditions before entering the scanner. Such exposure ensures the highest probability possible that the dogs will engage in the same cognitive functioning during scans presenting the same conditions.

Post hoc attributions of function to structure also pose a risk to the quality and validity of canine fMRI studies. Extrapolating a cognitive process from an area of activation (usually one that was unexpected) is known as reverse inference (Poldrack, 2006) and is better left for creating hypotheses for future research rather than making definitive conclusions. Poldrack (2006) suggests that reverse inference may hold ground if a margin of confidence and probability is used, such that areas that are activated by a large number of cognitive processes are given low levels of confidence when engaging in reverse inference, but areas that are activated by few processes are given a higher level of confidence. Henson 
(2006) explains that while reverse inference may not be the ideal way to look at data, it does lend itself to the identification of successful experimental replication and the ability to connect relating cognitive processes.

To further address the potential complications of reverse inference, researchers may make use of forward inference by seeking qualitatively different brain activations when comparing competing cognitive theories (Henson, 2006). That is, one can design an experiment with conditions that engage different cognitive processes according to one theory, but that do not in another theory. With this framework, the resulting activity patterns will be evidence for one theory. In a more general sense, contrasting a working hypothesis with a null hypothesis will allow more concise conclusions to be made from data obtained in functional imaging studies.

Methodological adjustments may also be suitable for improving the conceptual issue of inference. By considering the potential utility of pure insertion, researchers may enhance conclusions that differences in imaging data are due to the differences in experimental conditions, without the interference of confounding variables and extraneous sources of variance (Henson, 2006). This is especially important in studies with dogs, again due to the lack of feedback and explanation of strategies that exists in human research. Henson (2006) explains that when using pure insertion, if no variables other than those of experimental interest vary, then there is no reason to expect an underlying qualitative difference in brain activity. Thus, if the independent variable is precisely and singularly manipulated in studies of canine cognition using fMRI, then researchers may be better suited to make conclusions about canine brain structure-function relationships.

\section{Applications and Future Directions}

One of the most pressing applications of fMRI research with domestic dogs is the investigation of bio-behavioral markers of successful working dogs. Cobb, Branson, McGreevy, Lill, and Bennett (2015) define a working dog as one which is "operational in a private industry, government, assistant, or sporting context," while noting that these dogs may also simultaneously serve as human companions. Typically, these dogs fulfill roles in emotional support (service dogs) or in threat prevention (odor detection dogs). The working dog industry has been and is continuing to grow at a rapid rate, with dogs being trained in increasingly complex duties and the breeding programs producing greater numbers of puppies. Unfortunately, upwards of $50 \%$ of these dogs fail at some point in their training (e.g., Dalibard, 2009;
Maejima et al., 2007; Slabbert \& Odendaal, 1999; Wilsson \& Sinn, 2012), resulting in large-scale concerns of wasted funding, lost revenue, and a deficit in ethical considerations for individual dogs as transitions between working and pet dog roles can lead to stress and adoption difficulties.

While training programs and working purposes vary among working dog organizations, fMRI methodologies can be developed to identify common activation areas and patterns, as well as behavioral correlates, among dogs that pass rigorous training and succeed in the workforce. Discovering such bio-behavioral markers using cross-sectional and longitudinal designs over specific training histories may lead to better standards of identification, training, and treatment of dogs intended for working roles. An endophenotype for a specific working dog role may be developed in a stepwise fashion by using behavioral assessments to identify the most viable behavioral tendencies to fulfill the role and then correlating scores on such identifiers with brain activation data, such as neural responsiveness to target odors, auditory cues, or visual markers.

The use of fMRI, while in its infancy, may also bolster the reliability and validity of prior cognitive research with dogs. By adapting behavioral tasks for use in the scanner and/or correlating behavioral measures with in-scanner techniques, underlying cognitive processes may be better examined and evidenced. Such adaptation and correlation in human studies have preceded dog research, as the scientific community has successfully translated questions of human cognition historically targeted by self-report and behavioral measures into tasks to be completed in MRI. Such imaging capabilities have allowed simultaneous behavioral measurement of cognitive processes (i.e., the direct responding from the participant) and brain activation patterns that capture the once-illusive covert neural processing of cognitive tasks. Further, the convergence of multiple measures allows for thorough assessment of the strengths and weaknesses of individual cognitive theories. Building on the rise of cognitive neuroscience in human cognition, the merger of behavioral and neural responses by dog subjects will provide researchers with comprehensive and expansive data sets, and questions that were previously left to speculation may be explained in terms of neural structure and activation. Finally, the gamut of advanced analysis methods in human fMRI research, such as connectivity models (Jia, $\mathrm{Hu}$, et al., 2014) and multivariate pattern analysis and learning models (Deshpande, Libero, Sreenivasan, Deshpande, \& Kana, 2013) can be employed on dog fMRI data, potentially alleviating some of the issues with traditional activation models and giving us new insights into underlying neural mechanisms. 


\section{Comparative Mechanisms}

Comparisons between human and dog cognitive processes may be directly analyzed with the use of comparative fMRI methods (e.g., Andics et al., 2014). By presenting the same task to both humans and dogs, activation areas and patterns may be directly discussed. For example, questions of domestication and development of heterospecific social processing may target analogous neural structures and networks in dogs and humans over the individual lifespan. If one seeks to understand the similarities between emotion recognition in humans and dogs, then they may present both human and dog participants with the same stimulus set (e.g., humans smiling versus frowning) in the scanner and investigate neural activation patterns. This form of identical measurement eliminates common points of ambiguity in results due to methodology, such as differences in stimulus presentation and environment. With increased clarity of comparative measures between humans and dogs, investigations into the evolutionary development of neural structure and processes across species will be broader, more robust, and easier to implement. There is great potential to better understand which processes are due to convergent evolution or homology and the interaction between phenotype and ontology.

\section{Conclusions}

In summary, canine fMRI is a new and exciting frontier in comparative cognition research. The trainability of dogs, as well as their close social connection to humans, makes them a prime species for study in the MR scanner in an awake and unrestrained state. With continued refinement of methodology and conceptual ideas highlighting the utility of fMRI with dogs, we can expect to see an increase in the information we know about the function and structure of the canine mind. When embarking on a study of domestic dogs in fMRI, researchers must carefully consider the experimental design parameters, from subject to coil selection and from stimulus modality to presentation order. Conceptual issues should be addressed during experimental design, rather than post hoc, in order to ensure the viability of imaging data and the conclusions that are reached. With careful attention to each interlocking aspect of fMRI design, not only should the comparative cognition literature advance, but the neuroimaging literature as a whole should advance as well. To be sure, the recent combination of the "rise of the dogs" with neuroimaging has formed the foundation for the cognitive neuroscience of canine cognition.

\section{References}

Anaya García, M. S., Hernández Anaya, J. S., Marrufo Meléndez, O., Velázquez Ramírez, J. L., \& Palacios Aguiar, R. (2015). In Vivo study of cerebral white matter in the dog using diffusion tensor tractography. Veterinary Radiology \& Ultrasound, 56(2), 188-195. doi:10.1111/vru.12211

Andics, A., Gácsi, M., Faragó, T., Kis, A., \& Miklósi, Á. (2014). Voice-sensitive regions in the dog and human brain are revealed by comparative fMRI. Current Biology, 24(5), 574-578.

doi:10.1016/j.cub.2014.01.058

Bach, J.-P., Lüpke, M., Dziallas, P., Wefstaedt, P., Uppenkamp, S., Seifert, H., \& Nolte, I. (2013). Functional magnetic resonance imaging of the ascending stages of the auditory system in dogs. BMC Veterinary Research, 9, 210. doi:10.1186/1746-6148-9-210

Baker, M. A. (2013). Reduction of MRI acoustic noise achieved by manipulation of scan parameters: A study using veterinary MR sequences. Radiography, 19(1), 11-16. doi:10.1016/j.radi.2012.09.004

Baxi, M., Robinson, J. L., Katz, J., Waggoner, W., Beyers, R., Morrison, E.,... Deshpande, G. (Under review). Characterization of structural connectivity of the default mode network in dogs using diffusion tensor imaging. Scientific Reports, under review.

Becerra, L., Pendse, G., Chang, P. C., Bishop, J., \& Borsook, D. (2011). Robust reproducible resting state networks in the awake rodent brain. PLoS One, 6(10), e25701. doi:10.1371/journal.pone.0025701

Bensky, M. K., Gosling, S. D., \& Sinn, D. L. (2013). The world from a dog's point of view: A review and synthesis of dog cognition research. Advances in the Study of Animal Behavior, 45, 209-406. doi:10.1016/B978-0-12-407186-5.00005-7

Berns, G. S., Brooks, A. M., \& Spivak, M. (2012). Functional MRI in awake unrestrained dogs. PLoS ONE, 7(5), e38027. doi:10.1371/journal.pone.0038027 
Berns, G. S., Brooks, A., \& Spivak, M. (2013).

Replicability and heterogeneity of awake unrestrained canine fMRI responses. PLOS ONE, 8(12), e81698. doi:10.1371/journal.pone.0081698

Berns, G. S., Brooks, A. M., \& Spivak, M. (2015). Scent of the familiar: An fMRI study of canine brain responses to familiar and unfamiliar human and dog odors. Behavioural Processes, 110, 37-46. doi:10.1016/j.beproc.2014.02.011

Buckner, R. L., Andrews-Hanna, J. R., \& Schacter, D. L. (2008). The brain's default network: Anatomy, function, and relevance to disease. Annals of the New York Academy of Sciences, 1124, 1-38. doi:10.1196/annals.1440.011

Chen, G., Wang, F., \& Dillenburger, B. (2012). Functional magnetic resonance imaging of awake monkeys: Some approaches for improving imaging quality. Magnetic Resonance Imaging, 30(1), 36-47. doi:10.1016/j.mri.2011.09.010

Cobb, M., Branson, N., McGreevy, P., Lill, A., \& Bennett, P. (2015). The advent of canine performance science: Offering a sustainable future for working dogs. Behavioural Processes, 110, 96-104. doi:10.1016/j.beproc.2014.10.012

Cole, M. W., Yarkoni, T., Repovš, G., Anticevic, A., \& Braver, T. S. (2012). Global connectivity of prefrontal cortex predicts cognitive control and intelligence. The Journal of Neuroscience, 32(26), 8988-8999. doi:10.1523/JNEUROSCI.0536-12.2012

Cook, P. F., Spivak, M., \& Berns, G. S. (2014). One pair of hands is not like another: Caudate BOLD response in dogs depends on signal source and canine temperament. PeerJ, e596. doi:10.7717/peerj.596

Dalibard, G. H. (2009). Parameters influencing service dogs' quality of response to commands: Retrospective study of 71 dogs. Journal of Veterinary Behavior: Clinical Applications and Research, 4(1), 19-24. doi:10.1016/j.jveb.2008.08.008

Datta, R., Lee, J., Duda, J., Avants, B. B., Vite, C. H., Tseng, B.,... Aguirre, G. K. (2012). A digital atlas of the dog brain. PLoS ONE, 7(12), e52140. doi:10.1371/journal.pone.0052140
De Groof, G., Jonckers, E., Güntürkün, O., Denolf, P., Van Auderkerke, J., \& Van der Linden, A. (2013). Functional MRI and functional connectivity of the visual system of awake pigeons. Behavioural Brain Research, 239(1), 43-50. doi:10.1016/j.bbr.2012.10.044

Denison, R. N., Vu, A. T., Yacoub, E., Feinberg, D. A., \& Silver, M. A. (2014). Functional mapping of the magnocellular and parvocellular subdivisions of human LGN. NeuroImage, 102, 358-369. doi:10.1016/j.neuroimage.2014.07.019

Deshpande, G., Kerssens, C., Sebel, P. S., \& Hu, X. (2010). Altered local coherence in the default mode network due to sevoflurane anesthesia. Brain Research, 1318, 110-121. doi:10.1016/j.brainres.2009.12.075

Deshpande, G., Libero, L. E., Sreenivasan, K. R., Deshpande, H. D., \& Kana, R. K. (2013). Identification of neural connectivity signatures of autism using machine learning. Frontiers in Human Neuroscience, 7, 670. doi:10.3389/fnhum.2013.00670

Dilks, D. D., Cook, P., Weiller, S. K., Berns, H. P., Spivak, M. H., \& Berns, G. (2015). Awake fMRI reveals a specialized region in dog temporal cortex for face processing. PeerJ, e1115. doi:10.7717/peerj.1115

Duyn, J. H. (2012). The future of ultra-high field MRI and fMRI for study of the human brain. Neuroimage, 62(2), 1241-1248. doi:10.1016/j.neuroimage.2011.10.065

Fair, D. A., Cohen, A. L., Power, J. D., Dosenbach, N. U., Church, J. A., Miezin, F. M., ... Petersen, S. E. (2009). Functional brain networks develop from a "local to distributed" organization. PLoS Computational Biology, 5(5), e1000381. doi:10.1371/journal.pcbi.1000381

Feinberg, D. A., Moeller, S., Smith, S. M., Auerbach, E., Ramanna, S., Gunther, M.,... Yacoub, E. (2010). Multiplexed echo planar imaging for sub-second whole brain fMRI and fast diffusion imaging. PloS ONE, 5(12), e15710. doi:10.1371/journal.pone.0015710 
Golay, X., de Zwart, J. A., Ho, Y. C. L., \& Sitoh, Y. Y. (2004). Parallel imaging techniques in functional MRI. Topics in Magnetic Resonance Imaging, 15(4), 255-265. doi:10.1097/01.rmr.0000142829.79609.d4

Greicius, M. D., Kiviniemi, V., Tervonen, O., Vainionpää, V., Alahuhta, S., Reiss, A. L., \& Menon, V. (2008). Persistent default-mode network connectivity during light sedation. Human Brain Mapping, 29(7), 839-847. doi:10.1002/hbm.20537

Gross, B., Garcia-Tapia, D., Riedesel, E., Ellinwood, N. M., \& Jens, K. (2010). Normal canine brain maturation at magnetic resonance imaging. Veterinary Radiology and Ultrasound, 51(4), 361-373. doi:10.1111/j.1740-8261.2010.01681.x

Henson, R. N. (2004). Analysis of fMRI timeseries: Linear Time-Invariant models, event-related fMRI and optimal experimental design. In Frackowiak, Friston, Frith, Dolan, \& Price (Eds.), Human Brain Function, 2nd ed. (pp. 793-822). London: Elsevier.

Henson, R. (2006). Forward inference using functional neuroimaging: Dissociations versus associations. Trends in Cognitive Sciences, 10(2), 64-69. doi:10.1016/j.tics.2005.12.005

Hsu, Y., \& Serpell, J. A. (2003). Development and validation of a questionnaire for measuring behavior and temperament traits in pet dogs. Journal of the American Veterinary Medical Association, 223(9), 1293-1300. doi:10.2460/javma.2003.223.1293

Jacqmot, O., Van Thielen, B., Fierens, Y., Hammond, M., Willekens, I., Schuerbeek, P. V. (2013). Diffusion tensor imaging of white matter tracts in the dog brain. The Anatomical Record, 296, 340-349. doi:10.1002/ar.22638

Jia, H., Hu, X., \& Deshpande, G. (2014). Behavioral relevance of the dynamics of the functional brain connectome. Brain Connectivity, 4(9), 741-759. doi:10.1089/brain.2014.0300

Jia, H., Pustovyy, O. M., Waggoner, P., Beyers, R. J., Schumacher, J., Wildey, C., ... Deshpande, G. (2014). Functional MRI of the olfactory system in conscious dogs. PLoS ONE, 9(1), e86362. doi:10.1371/journal.pone.0086362
Jia, H., Pustovyy, O., Wang, Y., Waggoner, P., Beyers, R., Schumacher, J., ... Desphande, G. (2015). Enhancement of odor-induced activity in the canine brain by zinc nanoparticles: A functional MRI study in fully unrestrained conscious dogs. Chemical Senses. doi:10.1093/chemse/bjv054

King, J. A., Garelick, T. S., Brevard, M. E., Chen, W., Messenger, T. L., Duong, T. Q., \& Ferris, C. F. (2005). Procedure for minimizing stress for fMRI studies in conscious rats. Journal of Neuroscience Methods, 148(2), 154-160. doi:10.1016/j.jneumeth.2005.04.011

Kujawa, S. G., \& Liberman, M. C. (2009). Adding insult to injury: Cochlear nerve degeneration after "temporary" noise-induced hearing loss. The Journal of Neuroscience, 29(45), 14077-14085. doi:10.1523/JNEUROSCI.2845-09.2009

Kyathanahally, S. P., Jia, H., Pustovyy, O. M., Waggoner, P., Beyers, R., Schumacher, J., ... Deshpande, G. (2015). Anterior-posterior dissociation of the default mode network in dogs. Brain Structure and Function, 220(2), 1063-1076. doi:10.1007/s00429-013-0700-x

Lahti, K. M., Ferris, C. F., Li, F., Sotak, C. H., \& King, J. A. (1998). Imaging brain activity in conscious animals using functional MRI. Journal of Neuroscience Methods, 82(1), 75-83. doi:10.1016/S0165-0270(98)00037-5

Laird, A. R., Lancaster, J. L., \& Fox, P. T. (2005). BrainMap: The social evolution of a functional neuroimaging database. Neuroinformatics, 3, 65-78. doi:10.1385/NI:3:1:065

Lauer, A. M., El-Sharkawy, A. M. M., Kraitchman, D. L., \& Edelstein, W. A. (2012). MRI acoustic noise can harm experimental and companion animals. Journal of Magnetic Resonance Imaging, 36(3), 743-747. doi:10.1002/jmri.23653

Lu, H., \& van Zijl, P. C. (2012). A review of the development of Vascular-Space-Occupancy (VASO) fMRI. Neuroimage, 62(2), 736-742. doi:10.1016/j.neuroimage.2012.01.013 
Maclaren, J., Armstrong, B. S., Barrows, R. T., Danishad, K. A., Ernst, T., Foster, C. L., ... Zaitsev, M. (2012). Measurement and correction of microscopic head motion during magnetic resonance imaging of the brain. PLOS ONE 7(11): e48088 doi:10.1371/journal.pone.0048088

Maejima, M., Inoue-Murayama, M., Tonosaki, K., Matsuura, N., Kato, S., Saito, Y., ... Ito, S. (2007). Traits and genotypes may predict the successful training of drug detection dogs. Applied Animal Behaviour Science, 107, 287-298. doi:10.1016/j.applanim.2006.10.005

Mantini, D., Gerits, A., Nelissen, K., Durand, J. B., Joly, O., Simone, L., ... Vanduffel, W. (2011). Default mode of brain function in monkeys. The Journal of Neuroscience, 31(36), 12954-12962. doi:10.1523/JNEUROSCI.2318-11.2011

Martin, C., Martindale, J., Berwick, J., \& Mayhew, J. (2006). Investigating neural-hemodynamic coupling and the hemodynamic response function in the awake rat. Neuroimage, 32(1), 33-48. doi:10.1016/j.neuroimage.2006.02.021

Martín-Vaquero, P., da Costa, R. C., Echandi, R. L., Tosti, C. L., Knopp, M. V., \& Sammet, S. (2011). Magnetic resonance imaging of the canine brain at 3 and $7 \mathrm{~T}$. Veterinary Radiology \& Ultrasound, 52(1), 25-32. doi:10.1111/j.1740-8261.2010.01747.x

Miklósi, Á., \& Topál, J. (2013). What does it take to become 'best friends'? Evolutionary changes in canine social competence. Trends in Cognitive Sciences, 17, 287-294. doi:10.1016/j.tics.2013.04.005

Miller, E. K., Li, L., \& Desimone, R. (1991). A neural mechanism for working and recognition memory in inferior temporal cortex. Science, 254(5036), 13771379. doi:10.1126/science.1962197

Morgan, K. N., \& Tromborg, C. T. (2007). Sources of stress in captivity. Applied Animal Behaviour Science, 102(3-4), 262-302. doi:10.1016/j.applanim.2006.05.032
Nasrallah, F. A., Lee, E. L., \& Chuang, K. H. (2012). Optimization of flow-sensitive alternating inversion recovery (FAIR) for perfusion functional MRI of rodent brain. NMR in Biomedicine, 25(11), 12091216. doi: $10.1002 / \mathrm{nbm} .2790$

O’Craven, K. M., Rosen, B. R., Kwong, K. K., Treisman, A., \& Savoy, R. L. (1997). Voluntary attention modulates fMRI activity in human MT-MST. Neuron, 18(4), 591-598. doi:10.1016/S0896-6273(00)80300-1

Ogawa, S., Lee, T. M., Kay, A. R., \& Tank, D. W. (1990). Brain magnetic resonance imaging with contrast dependent on blood oxygenation. Proceedings of the National Academy of Sciences, 87(24), 9868-9872. doi:10.1073/pnas.87.24.9868

Ogawa, S., Tank, D. W., Menon, R., Ellermann, J. M., Kim, S. G., Merkle, H., \& Ugurbil, K. (1992). Intrinsic signal changes accompanying sensory stimulation: Functional brain mapping with magnetic resonance imaging. Proceedings of the National Academy of Sciences, 89(13), 5951-5955. doi:10.1073/pnas.89.13.5951

Petcharunpaisan, S., Ramalho, J., \& Castillo, M. (2010). Arterial spin labeling in neuroimaging. World Journal of Radiology, 2(10), 384. doi:10.4329/wjr.v2.i10.384

Poldrack, R. A. (2006). Can cognitive processes be inferred from neuroimaging data? Trends in Cognitive Sciences, 10(2), 59-63. doi:10.1016/j.tics.2005.12.004

Poldrack, R. A., Mumford, J. A., \& Nichols, T. E. (2011). Handbook of functional MRI data analysis. New York, NY: Cambridge University Press. doi:10.1017/cbo9780511895029

Power, J. D., Mitra, A., Laumann, T. O., Snyder, A. Z., Schlaggar, B. L., \& Petersen, S. E. (2014). Methods to detect, characterize, and remove motion artifact in resting state fMRI. Neuroimage, 84, 320-341. doi:10.1016/j.neuroimage.2013.08.048

Roberts, T., McGreevy, P., \& Valenzuela, M. (2010). Human induced rotation and reorganization of the brain of domestic dogs. PLoS ONE, 5(7), e11946. doi:10.1371/journal.pone.0011946 
Roth, G., \& Dicke, U. (2005). Evolution of the brain and intelligence. Trends in Cognitive Sciences, 9(5), 250-257. doi:10.1016/j.tics.2005.03.005

Satpute, A. B., Wager, T. D., Cohen-Adad, J., Bianciardi, M., Choi, J. K., Buhle, J. T., ... Barrett, L. F. (2013). Identification of discrete functional subregions of the human periaqueductal gray. Proceedings of the National Academy of Sciences, 110(42), 17101-17106. doi:10.1073/pnas.1306095110

Schafer, J. R., Kida, I., Xu, F., Rothman, D. L., \& Hyder, F. (2006). Reproducibility of odor maps by fMRI in rodents. Neuroimage, 31(3), 1238-1246. doi:10.1016/j.neuroimage.2005.12.060

Shellock, F. G., \& Crues, J. V. (2004). MR procedures: Biologic effects, safety, and patient care. Radiology, 232(3), 635-652. doi:10.1148/radiol.2323030830

Slabbert, J. M., \& Odendaal, J. S. J. (1999). Early prediction of adult police dog efficiency: A longitudinal study. Applied Animal Behaviour Science, 64, 269-288. doi:10.1016/S0168-1591(99)00038-6

Smith, J. A. (2010). Hazards, safety, and anesthetic considerations for magnetic resonance imaging. Topics in Companion Animal Medicine, 25(2), 98-106. doi:10.1053/j.tcam.2010.01.003

Snow, J. C., Pettypiece, C. E., McAdam, T. D., McLean, A. D., Stroman, P. W., Goodale, M. A., \& Culham, J. C. (2011). Bringing the real world into the fMRI scanner: Repetition effects for pictures versus real objects. Scientific Reports, 1, 130. doi:10.1038/srep00130

Spetch, M. L. (2010). Understanding how pictures are seen is important for comparative visual cognition. Comparative Cognition \& Behavior Reviews, 5, 163-166. doi:10.3819/ccbr.2010.50013

Su, M.-Y., Tapp, P. D., Vu, L., Chen, Y.-F., Chu, Y., Muggenburg, B.,...Head, E. (2005). A longitudinal study of brain morphometrics using serial magnetic resonance imaging analysis in a canine model of aging. Progress in Neuro-Psychopharmacology \& Biological Psychiatry, 29(3), 389-97. doi:10.1016/j.pnpbp.2004.12.005
Suthana, N. A., Donix, M., Wozny, D. R., Bazih, A., Jones, M., Heidemann, R. M., ... Bookheimer, S. Y. (2015). High-resolution 7T fMRI of human hippocampal subfields during associative learning. Journal of Cognitive Neuroscience, 27(6), 1194-206. doi:10.1162/jocn_a_00772

Thesen, S., Heid, O., Mueller, E., \& Schad, L. R. (2000). Prospective acquisition correction for head motion with image-based tracking for real-time fMRI. Magnetic Resonance in Medicine, 44(3), 457-465. doi:10.1002/1522-2594(200009)44:3 $<457:: A I D-M R M 17>3.0 . C O ; 2-R$

Todd, N., Josephs, O., Callaghan, M. F., Lutti, A., \& Weiskopf, N. (2015). Prospective motion correction of 3D echo-planar imaging data for functional MRI using optical tracking. NeuroImage, 113, 1-12. doi:10.1016/j.neuroimage.2015.03.013

Tóth, L., Gácsi, M., Miklósi, Á., Bogner, P., \& Repa, I. (2009). Awake dog brain magnetic resonance imaging. Journal of Veterinary Behavior Clinical Applications and Research, 4, 50. doi:10.1016/j.jveb.2008.09.021

Upadhyay, J., Baker, S. J., Chandran, P., Miller, L., Lee, Y., Marek, G. J., ... Day, M. (2011). Default-mode-like network activation in awake rodents. PloS ONE, 6(11), e27839. doi:10.1371/journal.pone.0027839

Venn, R. E., McBrearty, A. R., McKeegan, D., \& Penderis, J. (2014). The effect of magnetic resonance imaging noise on cochlear function in dogs. Veterinary Journal, 202(1). doi:10.1016/j.tvj1.2014.07.006

Wilsson, E., \& Sinn, D. L. (2012). Are there differences between behavioral measurement methods? A comparison of the predictive validity of two ratings methods in a working dog program. Applied Animal Behaviour Science, 141(3-4), 158-172. doi:10.1016/j.applanim.2012.08.012

Yacoub, E., Harel, N., \& Uğurbil, K. (2008). Highfield fMRI unveils orientation columns in humans. Proceedings of the National Academy of Sciences, 105(30), 10607-10612. 